Tam Nguyen

\section{Hintergrund}

Beim sogenannten Restless-LegsSyndrom (RLS) handelt es sich um eine neurologische, sensomotorische Störung, welche sich unter anderem durch Symptome wie Gefühlsstörungen und Bewegungsdrang in den Extremitäten äussert. Meist sind die Beine stärker betroffen als die Arme, und es kann neben den Empfindungsstörungen auch zu unwillkürlichen Bewegungen der betroffenen Körperpartien kommen. Folgende vier Symptome müssen für eine klinische Diagnose des RLS vorhanden sein:

- MissempfindungenmitBewegungsdrang;

- motorische Unruhe;

- verstärktes Auftreten der Beschwerden in Ruhephase und sofortige Verbesserung bei Bewegung;

- gehäuftes Auftreten der Symptome gegen Abend/Nacht.

Man unterscheidet zwischen der idiopathischen und der sekundären Form des RLS. Aktuelle Forschungsergebnisse deuten auf eine genetische Prädisposition für die idiopathische Form des RLS hin [1]. Zudem ist eine Antizipation zu beobachten, d.h., der Schweregrad der Krankheit nimmt über die Generationen zu und die Symptome treten in der Erbfolge schon früher auf [2]. Die sekundäre Form des RLSkann durch andere Störungen und Krankheiten (Urämie, Diabetes mellitus (DM), chronisch-venöse Insuffizienz (CVI), rheumatoide Arthritis, Polyneuropathie (PNP)) sowie Medikamente (Dopaminantagonisten und Antidepressiva) ausgelöst werden.

\title{
Restless-Legs-Syndrom: Fallbericht einer Therapie mit Padma 28
}

Mit einer Prävalenz von rund 7\% in Westeuropa und den USA (klinisch relevantes RLS: 2,7\%, Beschwerden häufiger als zwei Mal pro Woche) [3] ist das RLS eine der häufigsten neurologischen Krankheiten überhaupt. Die Ausprägung der Symptome erstreckt sich über einen weiten Bereich von «kaum bemerkbar» bis «schlafraubend». Da die Beeinträchtigung der Lebensqualität durch stärker ausgeprägte Symptome erheblich ist (Schlafmangel, Müdigkeit, Konzentrationsschwierigkeiten), suchen viele Betroffene Rat bei medizinischen Fachpersonen.

Das therapeutische Spektrum zur Behandlung des RLS umfasst neben der Behandlung einer zugrunde liegenden Krankheit auch das Absetzen möglicher triggernder Medikamente, den Verzicht auf Genussdrogen am Abend (Koffein, Nikotin, Alkohol), das Lösen kognitiver Aufgaben vor dem Schlafengehen sowie physiotherapeutische Übungen. Klassische pharmakotherapeutische Massnahmen bei RLS beinhalten Levodopa / DopaminAgonisten, Antiepileptika, Antiparkinsonika und Opiate, die allerdings alle mit einem entsprechend hohen Nebenwirkungspotenzial einhergehen. Viele Patienten sind trotz des hohen Leidensdrucks nicht gewillt, sich einer solchen Therapie zu unterziehen. Die Nachfrage und der Wunsch nach alternativen Behandlungsoptionen sind deshalb gross.

\section{Therapieansatz mit Padma 28}

Padma 28 ist ein pflanzliches Arzneimittel, das in der Schweiz nach den Prinzipien der Tibetischen Medizin hergestellt wird (Swissmedic Nr. 58436, identisch mit dem kassenzulässigen Padmed Circosan, Swissmedic Nr. 60131). Es wird eingesetzt bei Parästhesien wie Kribbeln, Ameisenlaufen, Schwere- und Spannungsgefühl in den Beinen und Armen, Einschlafen von Händen und Füssen sowie Wadenkrämpfen. Diese Symptome können sowohl durch arterielle Durchblutungsstörungen als auch durch eine gestörte venöse Mikrozirkulation im Gewebe verursacht werden. Verschiedene Fallberichte lassen darauf schliessen, dass Padma 28 bei vielen Grunderkrankungen des sekundären RLS wie z.B. DM Typ 2 [4] sowie Symptomen von CVI [5] und PNP [6] unterstützend eingesetzt werden und die Beschwerden und damit auch die Lebensqualität der Betroffenen erheblich verbessern kann. Für RLS-Patienten, die sich komplementär- und phytomedizinische Alternativen wünschen, eröffnet dies neue Therapiemöglichkeiten in der Behandlung der Grunderkrankungen und des damit einhergehenden RLS.

\section{Fallbericht}

\section{Patientin}

Die Patientin war bei Therapiebeginn (März 2014) 30 Jahre alt und hatte ein klinisch relevantes RLS mit den vier eingangs erwähnten Kardinalsymptomen. Die damit verbundenen

\section{KARGER}

() 2017 S. Karger GmbH, Freiburg

Fax +497614520714
Dr. med. Tam Nguyen

Santémed Gesundheitszentrum Winterthur

Gertrudstrasse 1, 8400 Winterthur, Schweiz

tam.nguyen@santemed.ch 


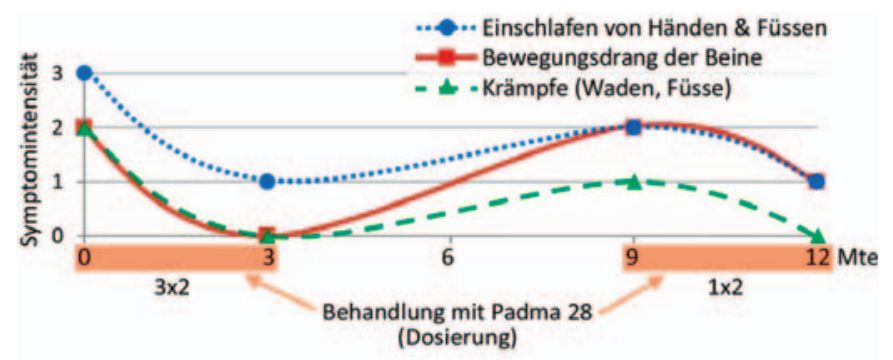

Abb. 1. Verlauf der Ausprägung der fünf beschriebenen Symptome und deutliche Abnahme der Intensität während der beiden Behandlungsphasen.

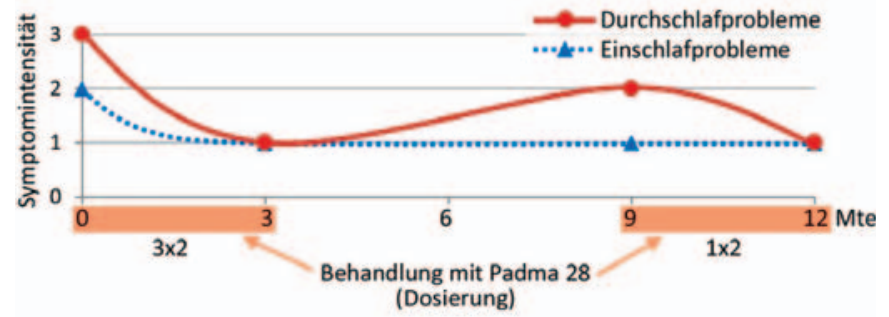

Symptomintensität: $0=$ beschwerdefrei $\quad 1=$ schwach $\quad 2=$ mittel $\quad 3=$ stark
Beschwerden hatten sich nach einer Diskushernie in den Lendenwirbeln (zwischen L4 und L5) im Jahr 2007 entwickelt. Die medizinische Vorgeschichte beschränkte sich auf den gelegentlichen Konsum von Genussdrogen (Alkohol und Koffein) sowie einen Nikotinabusus vom 15. bis zum 30. Lebensjahr (5 pack years). Die Patientin nahm keine weiteren Medikamente ein. Die Familienanamnese zeigte, dass wahrscheinlich keine idiopathische, sondern eine sekundäre Form des RLS vorliegt, deren Ursprung vermutlich die Diskushernie von 2007 ist.

\section{Therapie und Verlauf}

Die Patientin befand sich vor Therapiebeginn nicht bezüglich RLS in Behandlung. Aufgrund ihrer Affinität zur Phytotherapie setzte sie das pflanzliche Arzneimittel Padma 28 ein, und zwar in einer Dosierung von $3 \times 2$ Kapseln/Tag (morgens/mittags/abends). Unter dieser Behandlung nahmen die Beschwerden im Lauf von 3 Monaten deutlich ab (Abb. 1). Während sich das Symptom Einschlafen von Händen und Füssen von starker auf eine schwache Ausprägung reduzierte, verschwanden der Bewegungsdrang der Beine und die Krämpfe in den Waden und Füssen vollständig (anfangs mittelstarke Ausprägung). Gleichzeitig reduzierten sich auch die Ein- und Durchschlafstörungen (von mittelstark bzw. stark auf schwach). Wegen dieser deutlichen Verbesserung setzte sie das Arzneimittel nach 3 Monaten ab.

Im Lauf der nächsten 6 Monate verstärkten sich die Beschwerden nach und nach wieder etwas und die Therapie mit Padma 28 wurde auf ärztliche Verschreibung in der Dosierung $1 \times 2$ Kapseln/Tag wieder aufgenommen. Im weiteren Verlauf reduzierte sich die Ausprägung der Beschwerden wieder auf eine schwache Intensität; die
Krämpfe in den Waden und Füssen verschwanden wiederum ganz (Abb. 1). Die Therapie wird in der Erhaltungsdosis von $1 \times 2$ Kapseln täglich weitergeführt.

Gemäss Angaben der Patientin wirkte sich körperliche Aktivität (Pilates) jeweils positiv, Stress und längere Immobilität (z.B. bei Flugreisen) hingegen negativ auf die Symptomintensität aus. Ihre Blutwerte bei $\mathrm{t}=6$ Monate zeigten keinerlei Auffälligkeiten. Ebenfalls bei $t=6$ Monate gab die Patientin aus eigener Motivation den Nikotinabusus auf, was sich aus ihrer Sicht positiv auf das Einschlafen von Händen und Füssen auswirkte.

\section{Schlussfolgerung}

Padma 28 bietet mit seiner Wirksamkeit bei Durchblutungs- und Empfindungsstörungen eine geeignete Therapieoption bei RLS - insbesondere auch, wenn von den Betroffenen komplementäre oder pflanzliche Therapien gewünscht werden oder die Standardtherapie mit Antiepileptika und Opiaten aufgrund des hohen Nebenwirkungspotenzials vermieden werden soll oder nicht vertragen wird.

Für die Symptome «Einschlafen von Händen und Füssen», «Bewegungsdrang der Beine», «Durchschlafprobleme» und «Krämpfe in Waden und Füssen» ist eine deutliche Korrelation des Verlaufs der Intensität und den Phasen der Einnahme des Arzneimittels zu sehen. Dies deutet darauf hin, dass das Therapieschema $(3 \times 2$ Kapseln/Tag Initialdosis, bis sich Besserung einstellt, dann $1 \times 2$ Kapseln/ Tag Erhaltungsdosis) auch bei diesem Krankheitsbild bewährt.

\section{Literatur}

1 Winkelmann J, Schormair B, Lichtner P, et al: Genome-wide association study of restless legs syndrome identifies common variants in three genomic regions. Nat Genet 2007;8:1000-1006.

$2 \mathrm{Lu} \mathrm{Y:} \mathrm{Genetics} \mathrm{of} \mathrm{Restless} \mathrm{Legs} \mathrm{Syndrome:}$ Mutational Analysis of Candidate Genes at the RLS5 Locus in a German Family. Med. Dissertation. Kiel, 2007.

3 Allen RP, Walters AS, Montplaisir J, Hening W, Myers A, Bell TJ, Ferini-Strambi L: Restless legs syndrome prevalence and impact: REST general population study. Arch Intern Med 2005;165:1286-1292.

4 von Muralt Portmann D: Parästhesien bei Diabetes mellitus: Therapieansätze mit Padma 28. Schweiz Z Ganzheitsmed 2010;22:209-210.
5 Locher A: Padma 28 bei venösen Beinbeschwerden - ein Fallbericht. Schweiz Z Ganzheitsmed 2012;24:272-273.

6 Rüttgers JO: Polyneuropathie: Therapie mit Padma 28 - Wirksamkeit eines tibetischen Vielstoffgemisches. Schweiz Z Ganzheitsmed 2014;26:146-148. 